Article

\title{
Functional Information: Towards Synthesis of Biosemiotics and Cybernetics
}

\author{
Alexei A. Sharov \\ National Institute on Aging, 251 Bayview Boulevard, Baltimore, MD 21224, USA; \\ E-Mail: sharoval@mail.nih.gov \\ Received: 10 March 2010; in revised form: 6 April 2010 / Accepted: 21 April 2010 /
}

Published: 27 April 2010

\begin{abstract}
Biosemiotics and cybernetics are closely related, yet they are separated by the boundary between life and non-life: biosemiotics is focused on living organisms, whereas cybernetics is applied mostly to non-living artificial devices. However, both classes of systems are agents that perform functions necessary for reaching their goals. I propose to shift the focus of biosemiotics from living organisms to agents in general, which all belong to a pragmasphere or functional universe. Agents should be considered in the context of their hierarchy and origin because their semiosis can be inherited or induced by higher-level agents. To preserve and disseminate their functions, agents use functional information - a set of signs that encode and control their functions. It includes stable memory signs, transient messengers, and natural signs. The origin and evolution of functional information is discussed in terms of transitions between vegetative, animal, and social levels of semiosis, defined by Kull. Vegetative semiosis differs substantially from higher levels of semiosis, because signs are recognized and interpreted via direct code-based matching and are not associated with ideal representations of objects. Thus, I consider a separate classification of signs at the vegetative level that includes proto-icons, proto-indexes, and proto-symbols. Animal and social semiosis are based on classification, and modeling of objects, which represent the knowledge of agents about their body (Innenwelt) and environment (Umwelt).
\end{abstract}

Keywords: functional information; biosemiotics; cybernetics; evolution; agent; origin of life; Umwelt; learning; biological complexity; biological signal processing

PACS Codes: 87.18.-h, 87.85.Ng 


\section{Introduction}

One of the major revelations of the 20th century is that life is intrinsically related to information processing and communication. The existence of each species depends on the transfer of genetic messages from parents to offspring [1]. Also, the survival and reproduction of individual organisms depends on their ability to model their environment and use these models to optimize their functions [2]. Understanding of the informational nature of life has led to the emergence of two new disciplines, cybernetics and biosemiotics, which approached the same problem from different angles. Cybernetics was conceived to study communication and control in machines and living organisms in the light of the information theory [3,4]. However, because living organisms are too complex and we still have very limited means to control them, cybernetics has much stronger links with technology (i.e., communication industry, computers, and robots) than with biology. Computers can process user-supplied information without "understanding" the problem that they help to solve. Thus, the algorithms of information processing appeared more important for cybernetics than the meaning of information. In contrast, biosemiotics is focused on studying living organisms and their unique ability to generate and interpret meaningful information [5]. Thus, biosemiotics and cybernetics appeared to be separated by the boundary between life and non-life.

But both disciplines can benefit from their integration in our development of technology. As artificial agents become more complex, they resemble living organisms and require novel principles for their design and analysis that go beyond traditional cybernetic approaches (e.g., goal oriented feedback, attractors, and computation). In particular, to achieve their goals, complex agents need efficient internal communication (between autonomous subagents, and with their future states) as well as external communication with other agents. Communication includes production and interpretation of signs, therefore analysis of complex agents requires semiotic approaches. Combined cybernetic-biosemiotic methodology can be applied also to the management of human knowledge, which now evolves towards multiplicity of underlying models and integration of heterogeneous data [6]. Analysis of cellular information processing networks can help to design flexible multi-purpose knowledge repositories.

This paper follows previous attempts to bridge the gap between biosemiotics and cybernetics, including second-order cybernetics [7,8], theory of autopoiesis [9], artificial life [10], evolutionary semiotics [11,12], organic codes [13], and cybersemiotics [14]. As one reason for his construction of a cybersemiotics Brier argues that information (as it is understood in cybernetics) is not enough to explain the phenomena of experience, communication, and knowledge [14]. He suggested to complement it with the semiotic theory and philosophy of Charles Peirce [15], which is based on the distinction between three basic categories: Firstness (chaos, potentiality, pure feeling and potential qualia), Secondness (force, will), and Thirdness (habits, symbols).

However, many obstacles remain on the path of integration. The notion that natural and artificial agents should be analyzed within the same logical framework is still not fully accepted in biosemiotics. The idea that life and semiosis are coextensive [5,12] is often interpreted literally, so that artificial agents appear non-semiotic. Another challenge comes from molecular biology which studies complex molecular machines involved in DNA replication, transcription, and protein synthesis. Molecular machines have no learning capacity, and in this respect they are similar to human machines. However, it is not logical to exclude them from the consideration of biosemiotics because they participate in 
cellular semiosis [13]. All agents are externally programmed (e.g., by genome, family, culture); and these external programs that carry historical and evolutionary roots appear more important for agents than the presence of individually acquired programs. Learning often requires longer times than the life span of individual agents, thus it should be viewed as an optional rather than necessary feature of agents. The role of agents in generating and processing of information is not fully recognized within the field of cybernetics, which is another obstacle for a synthesis between cybernetics and biosemiotics [11]. Information is often considered as a physical property of material objects [16], as a method in the theory of probability [17], or as input and output of computation [18]. Finally, analysis of agents requires an update for the philosophy of science. Agents are subjective beings and therefore we need to find a place for subjectivity within the scientific worldview. Based on Peirce's semiotic philosophy Brier considers that the universe has some very basic elements of consciousness such as pure feelings, wills, and a tendency to take habits [14]. However, his approach is not compatible with biology as a science. Development of habits in living organisms requires heredity or long-term memory which has not been found in the physical universe, and feelings require complex heritable sensors which are also not found outside of life and its products.

In this paper I follow a functional-evolutionary approach to agents in general, which are defined as systems with goal-directed programmed behavior. Agents are either living organisms or their products because only these systems are known to pursue their goals. Agents are interconnected horizontally, hierarchically, and genealogically; they often include subagents and are always produced by other agents of comparable or higher complexity. Agents can be well individuated or diffused ("swarm agents"), autopoietic, autotrophic, with or without learning capacity. What unites them, is their ability to perform functions for the purpose of reaching certain goals. Functions of agents are encoded and controlled by a set of signs which I call functional information. These include stable memory signs, transient messengers, and natural signs. Agents always receive some of their functional information from parental/recruiter agents and often follow parental/recruiter goals. This induced semiosis is common for living organisms and artificial devices. In contrast to the cybernetic understanding of agents with its emphasis on control, feedback loops, and attractors, my approach is focused on the origin, evolution, functionality, and communication of agents.

The origin and evolution of functional information is discussed in terms of transitions between vegetative, animal, and social levels of semiosis [19]. Vegetative semiosis is based on code-based mapping of sign molecules, which encode and control basic cellular functions. The origin of life is seen as the emergence of autocatalytic molecules that can encode properties of a larger host system in a way that enhances autocatalysis. Such a system is an agent because it can be described in terms of encoded goal-directed actions, although an alternative mechanistic description is also possible. But mechanistic models cannot fully capture the dynamics of complex agents; instead they can be applied only to their simple components. Agents represent a new cross-disciplinary ontological entity because we can describe them in terms of actions, signs, goals, and benefits, which do not belong to the vocabulary of physics. Simple agents belong to the "gray" transitional zone as they can be described both semiotically and mechanically. Higher levels of semiosis (animal and social) are grounded in the vegetative level and include classification and modeling of objects, which represent the knowledge of an agent about itself and its environment, i.e., Innenwelt and Umwelt [2]. In addition, advanced agents develop logic 
that helps them to produce new sequences of actions and new models of the world that are more likely to be successful than random actions and random models. Because most artificial devices are not yet capable of learning and evolution, their functional information has human origin. However, future artificial agents may have increased abilities to generate their own functional information, and some of them (synthetic organisms) may be capable of autonomous adaptive evolution.

\section{Agents}

Contemporary biology is based mostly on the material understanding of life: cells and organisms are described in terms of parts (e.g., proteins, lipids, nucleic acids, carbohydrates) and their interactions. An alternative "relational" approach, is to define living systems on the basis of their functions rather than composition [20,21]. If an artificial device performs the same (or similar) functions as a living organism, then, according to relational biology, it is alive. However, it would be confusing to apply the term "living organism" to artificial devices. Instead, it is better to use the term "agent" which equally fits to living organisms and artificial devices. Here I consider an agent as a system with spontaneous activity whose actions are programmed for reaching certain goals. Goals are considered in a broad sense, including both achievable events (e.g., capturing a resource or producing an offspring), and sustained values (e.g., survival, energy balance, and attention). Goals of autopoietic systems include production of functional body parts during development, survival, and reproduction [9]. The notion of agent is more complex than anything studied in physics and cannot be defined solely in terms of composition and relations. Thus, its definition includes notions of "action" and "goal", which do not belong to the vocabulary of physics.

Being an agent is not a physical property, which implies that agents are not universally identifiable. We can learn to recognize specific kinds of agents, but there is no rule to identify any kind of agent. Humans and other animals learn that agents are unusual and often dangerous beings. They are unpredictable, adaptive, and can be aggressive. But humans often make mistakes in identification of agents. Celestial objects were erroneously thought to be agents (gods). Also, people never expected that their body is made of smaller sub-agents until the emergence of cellular biology. Scientists still do not have a consensus on whether viruses and individual genes are agents or not. The following three criteria can help us to distinguish agents: (1) agents select specific actions out of multiple options, (2) these selected actions are useful in a sense that they help agents to reach their goals, and (3) agents do not emerge by chance, they are produced only by other agents of comparable or higher level of functional complexity [22]. These criteria, however, are difficult to apply. Agents may remain dormant for a long time; thus, actions are not instantly detectable. Actions may occur at the molecular level and therefore go unnoticed if we don't use molecular sensors. Evaluation of benefits is also not trivial because we do not know the goals of agents. We usually assume that goals of living organisms include survival and reproduction, a notion that comes from the theory of genetic selection. However, an organism may follow a goal of a larger agent (e.g., family or population) and sacrifice its life for the benefits of the super-agent. Actions may include multiple steps and only the last step brings benefits; thus, it is difficult to understand the benefits of previous steps. Because of these obstacles, it may be difficult to prove that some system in NOT an agent. Thus, I suggest following a conservative scientific approach and apply the term "agent" only to those systems that are well proven to have reproducible 
goal-directed activities. In particular, I do not consider the existence of non-material agents (gods) and potential agency in immortal systems (e.g., in the universe). Mortality implies that agents are either self-reproducing systems or products of other self-reproducing systems. This is a testable necessary condition which helps to narrow down the set of systems that can be agents.

Goals may emerge within the agent, or alternatively can be set by parental agents or higher-level agents. Primitive organisms mostly follow genetically inherited goals, thus, their individual contribution to these goals is relatively small. In contrast, higher animals are capable to develop novel individual goals, especially those that are related to short-term needs (e.g., fast response, ability to find resources and avoid enemies). In human populations, goals are communicated and propagated through the cultures. Goals of artificial agents are usually set by their designers; however, advanced robots can learn and develop new lower-level goals that help to reach externally supplied goal of the higher level. Learning capacity is optional for agents, but non-learning agents always originate from learning agents and are supplied with previously developed tools and programs that are necessary for performing their functions.

Agents often have a hierarchical structure and contain subagents. For example, multicellular organisms are made of cells and products of their activity, and cells are made of smaller subagents like organelles and chromosomes. Hoffmeyer referred to this nested structure as "boundaries within boundaries" [23]. Some agents have no boundary at all and are represented by free subagents united by communication or common origin (e.g., families, dems, populations, species, and communities). These agents can be called swarm agents, following the terminology of [23]. Subagents in swarms may interact and exchange information, however it is conceivable that some swarms are united solely by their common origin (i.e., common information and goals).

Because agents do not emerge by chance, they persist in the world only via continuous production of other agents. All agents are artifacts because they are manufactured by other agents, which matches the notion that "life is artifact making" [24]. Living agents are capable of self-repair and self-reproduction, a property known as autopoiesis [9]. But autopoietic agents may still depend on the environment and available resources. For example, predators need prey to survive, parasites need host organisms, and computer viruses propagate only within a network of interconnected computers. Only autotrophic agents can make all necessary parts from resources that are not produced by other agents, and therefore they are fully independent. All autotrophic agents are autopoietic, but not all autopoietic agents are autotrophic. Agents can be semi-autopoietic if they repair only certain components of their body but not capable to self-reproduction. Worker bees and mules are examples of semi-autopoietic agents; they can repair their cells and tissues but they cannot reproduce. Similarly, somatic cells within a multicellular living organism are not fully autopoietic. They have lost their ability of infinite selfreproduction, and some even lost their nucleus (erythrocytes). In contrast, germ cells (gametes and their progenitors) are fully autopoietic and potentially immortal.

Functional complexity of new agents is always comparable or lower than the complexity of parental agents because new functions appear only by modifications of already existing functions. Thus, the complexity can increase in lineages of agents only slowly by gradual modification of already existing functions. As a result, the rate of progressive evolution is limited, whereas regressive evolution can be fast. This limitation on the rate of progressive evolution is known as a principle of gradualism in 
evolution [22]. Gradualism was criticized based on the facts from genetics and paleontology. Many mutations produce instant big changes of the phenotype. Paleontological records also show rapid changes of phenotypes in numerous lineages during very short transitional times between stable epochs, which prompted Gould and Eldredge to develop the concept of punctuated equilibrium [25]. Dawkins argued that the discreteness of paleontological records is compatible with gradualism because it resulted mostly from migration and propagation of already existing species, rather than from suddenly accelerated evolutionary process as claimed by Gould [26]. However, the existence of macro-mutations and increased variation following environmental changes are still difficult to reconcile with gradualism. To avoid criticism, the principle of gradualism was reformulated in terms of complexity rather than morphology [22]. Morphology is the tip of the iceberg of evolutionary changes, which mostly occur at the molecular level. Species have developed molecular mechanisms that can support rapid morphological changes in stress conditions. These modifications do not change the total complexity of the system, they simply convert physiological and molecular complexity into new morphology. According to this new understanding of gradualism, the total functional complexity can increase only gradually, whereas certain morphological and physiological characteristics of organisms may change much faster [22].

The principle of gradualism does not contradict to the idea that complex systems show emergent behaviors, which is an important property of life [27]. It only restricts the rate of emergence by stating that new functions do not appear instantly but emerge gradually via modification of already existing functions. Simple modifications can be done fast enough but multi-component modifications take longer times. The more complex are the agents, the faster is their rate of developing novel functions because of several positive feedback mechanisms [28]. First, functions cooperate, so that better performance in one function may help to develop another function. Second, functions duplicate and get specialized. Finally, existing functions include manufacturing of specific tools or organs which need maintenance and recycling, therefore they create niches for novel maintenance functions. In evaluating the rate of emergence, it is important to distinguish the appearance of true novel functions from the activation of already existing silent functions. For example, a fertilized egg has very few active functions, however it carries the encoded capacity to perform many other functions that are going to be activated at certain developmental stages. Thus, the principle of gradualism refers to the full functional capacity of an agent, rather than to the set of currently active functions.

Because living systems are substantially more complex than non-living natural systems, the statement "life from life" follows from the principle of gradualism. In particular, complex systems cannot originate by pure chance, and life gradually emerged from very simple primordial systems. The statement "life from life" can be further generalized as "agents from agents" because the principle of gradualism works for all kinds of agents. Because living organisms appeared before human-made agents, all agents originate from life. Thus, the world of all agents, which I propose to call pragmasphere, is an extension of the biosphere. The distinction between natural and artificial appears less important than the distinction between agents and non-agents. The notion of pragmasphere thus becomes the meeting point of cybernetics and biosemiotics. However, the synthesis of these disciplines would require a revision of terminology and basic assumptions. It should be recognized that any agent is more than a physical body but a link between its parental agents and potential future products. 
Programmed artificial devices may be not enough smart and lack learning abilities, but they are manufactured by humans and produce useful things. Thus, they are components of human functional cycles and evolve together with human knowledge. To facilitate the synthesis of biosemiotics and cybernetics we need to apply principles of biosemiotics to all agents and not to restrict them to living organisms.

\section{Functions}

Function is a reproducible sequence of actions, which is beneficial for the agent. Reproducibility implies that functions are always encoded and controlled by signs, as discussed in section 4 . Living organisms have specific sets of functions at each level of their organization. The most simple are molecular functions, which are well described by the Gene Ontology [29]. The simplest ones usually fall into categories of binding and catalysis. Molecular functions are performed by specialized molecular sub-agents. For example, the DNA-polymerase complex duplicates the DNA molecule; and ribosomes synthesize proteins on the basis of the input mRNA sequence. Proteins often have several domains specialized for different functions, which are thus combined into a complex function with multiple actions. For example, the P300 protein is responsible for activation of transcription via acetylation of histones in the promoter region and possible interactions with the transcription initiation complex [30]. This protein has at least 8 domains, including a HAT domain to modify histones, a bromodomain to recognize histone modifications, a KIX domain to interact with CREB transcription factor, zinc-finger domains for DNA binding, and a coactivator of nuclear receptors. Obviously, P300 plays the role of an information hub, where multiple molecular signals are integrated to control the expression of the gene.

Input-output relations of molecular agents are often described as "information processing" because DNA replication is similar to string copying, and protein synthesis resembles computation. However, this metaphor may be misleading. Processes of DNA replication and protein synthesis taken alone (e.g., in vitro) have nothing to do with information because molecules of nucleic acids are processed as material objects irrespective of their meaning. However, in the context of a cell, these actions indeed represent information processing. Reductionism attempts to attribute all the functions of organisms to the agents at the lowest hierarchical level (i.e., to molecular complexes). This approach ignores the benefits of functions, which usually appear only at higher hierarchical levels. For example, DNA repair and proofreading are performed by certain protein complexes within a cell, but the benefits from these processes go far beyond the life of one organism. These benefits can be fully accounted for only in long lineages that include thousands of generations.

Functions at the cellular level include resource capturing, growth, metabolism, modification of the cytoskeleton, and surface properties, sensing of external conditions, cell cycle, and control of major internal processes. Each of these functions requires thousands and millions of molecular interactions. Multicellular organisms have even more complex sets of functions related to differentiation of cells. Each type of cells becomes specialized in performing unique functions that are necessary for the entire organism. Functions of the whole organism include eating, digestion, excretion, sensing, movement, mating, and reproduction. Finally, there are functions at the level of super-organisms (e.g., families, populations, species, and ecosystems). Functions of a colony include communication (bee dances), 
construction of nests, and defense (soldier ants). Ecosystems also can be viewed as agents, but their integrity is rather weak because many populations can easily migrate in and out. Examples of ecosystem functions are carbon circulation and recycling of dead organisms.

Agents often outsource their functions to server agents, which can be either manufactured or recruited. Sever agents have induced semiosis because their goals and functional information (defined in the next section) are modified or reset by master agents. The set of all server agents represents a functional envelope for master agents. Human functional envelope includes manufactured machines as well as recruited living organisms (cultivated plants and domesticated animals). Making functional envelopes is not specific to humans, it is rather a standard strategy for all agents. Animal body (somatic cells) is a mortal functional envelope for immortal germ cells. Similarly, a bacterial cell can be viewed as a functional envelope for the DNA molecule. Production of worker bees by a bee queen is another example of a functional envelope. Recruitment of other agents is also a widespread strategy in nature. Fungi in lichens recruit unicellular algae for photosynthesis and production of nutrients [31]. Leaf-cutting ants and bark beetles are farming fungus [32,33]. Development of functional envelopes is more than just increasing the number of agents; it creates super-agents that belongs to a new level of agent hierarchy. This process was called "metasystem transition" by Turchin [34], and "major transition" by Maynard Smith and Szathmáry [35].

\section{Functional Information}

Any function of an agent has to be reproducible, which means that agents should be able to repeat corresponding actions with a certain fidelity to ensure the same beneficial result. The only way to ensure this reproducibility over long evolutionary times is to manufacture, preserve, and replicate signs that control actions; thus, every function is encoded and controlled by signs. Storing signs in memory (e.g., genetic, epigenetic, or neural) can be interpreted as self-communication, because memory is a message sent by an agent to its own future state. The purpose of self-communication is to transfer the ability to perform functions, so that the agent preserves its functionality. The genome can be seen as a long-term "memory", which extends over many generations [19,36]. Transfer of the genome to the progeny is a form of self-communication because progeny is a continuation of the parent self [36]. Because signs deteriorate with time, they can persist indefinitely only by replication (copying, duplication). Thus, long-term memory signs have to be both copied and interpreted, which is known as a principle of sign duality [37]. Besides stable memory-signs, functions are also controlled by transient messengers generated by sensors or logical devices. Messengers are produced in response to certain external or internal conditions and help to optimize the timing of actions.

Signs are often defined based on their semantics, i.e., the ability to stand for something else for somebody [38]. Similarly, Peirce considered sign as a triadic relation between a sign vehicle, an object which it stands for, and interpretant which is induced by the sign in the interpreter [15]. Because I am interested in the role of signs in supporting the functions of agents, I prefer to emphasizes the pragmatic aspect of signs, i.e., their ability to specify or modify actions of agents in a beneficial way [39]. In other words, agents use signs to organize their activity. Activity of agents may result in the production of other signs (e.g., mRNA is synthesized using DNA as a template), or in the production of agent components (e.g., resources, structural elements, or sub-agents). Being a sign is not a physical 
property, instead it is a semiotic property. For example, a DNA molecule taken alone is just a chemical; but it can play the role of a sign for some living organism. Theoretically, any material object can be a sign for some agent who can interpret it in functional terms. However, most important are those signs that are produced by agents for communication purposes (including self-communication).

Semiotics and cybernetics historically use different terminology. The central notion of semiotics is "sign" whereas cybernetics uses the term "information". The term "information" is often used in a narrow sense as a degree of non-randomness or negentropy [17] or as a sequence of characters in the text or in DNA [24], however discussion of these meanings will carry us away from the topic of the paper. Instead, I will follow the definition of Bateson that "information - the elementary unit of information - is a difference which makes a difference" [40]. This understanding of "information" brings it close to the notion of "sign", however these terms are still not synonymous. Besides other differences in their meanings, I would like to emphasize the aggregative nature of information which unites many signs used together by an agent. In human semiotics, the notion of "sign" is often applied to individual words or sentences, for which the meaning can be clearly specified, but not to a dictionary, library, or database. In contrast, the term "information" is relevant for large and heterogeneous sets of signs. Thus, I consider information as a sign or set of signs used together by agents. To emphasize the functional role of information I proposed the term "functional information" which is a set of signs that encode the functions of the organism [36]. In this paper I want to widen the meaning of this term and include also those signs that control the functions. Functional information of organisms includes their genome, epigenome, internal messengers (e.g., mRNA, miRNA, transcription factors, kinases, and phosphatases), external messengers (e.g., pheromones), and natural signs (e.g., temperature and salinity of water). For comparison, Barbieri proposed the term "organic information" which is applied in a narrow sense to replicated sequences only [24]. The notion of "functional information" can be easily extended from organisms to artificial agents. Then, semiosis can be defined as a set of processes by which functional information is interpreted, duplicated, modified, and disseminated by agents for their own benefits.

\section{Vegetative Semiosis: Emergence and Early Evolution of Functional Information}

Functional information evolved in parallel with living organisms starting from the origin of life, and primitive agents differ substantially from advanced agents in the level of complexity of their semiotic processes. Thus it is important to delineate threshold zones that separate primitive levels of semiosis from advanced levels [19]. To separate semiotic systems from non-semiotic, Kull used the functional cycle of Uexküll as a basic model of semiosis. Then he proposed to distinguish between vegetative, animal, and cultural levels of semiosis, ordered by increasing complexity. He also argued that these three levels of semiosis can be associated with three types of signs defined by Peirce: icons, indexes, and symbols, respectively. Although I agree with Kull on the distinction between vegetative, animal, and cultural levels of semiosis as major transitions in the evolution of life, it is debatable if these levels can be associated with icons, indexes, and symbols, respectively. Instead, I apply Peirce's terminology only for animal and cultural semiotic levels, and use a modified classification of signs for vegetative semiosis (Figure 1). Another attempt to differentiate between levels of semiosis is the pragmatic scale of "consciousness" (ConsScale) which was applied to both living and artificial agents [41]. Based on 
the relationship between the body, receptors, action machinery, sensorimotor coordination, and memory, agents were classified as reactive, adaptive, attentional, executive, emotional, self-conscious, empathyc, social, human-like, and super-conscious. Animal semiosis, as defined by Kull, apparently starts from the attentional level. The weakness of ConsScale is that it does not consider the semiotic functions of agents (production, dissemination, and interpretation of signs), and agent behaviors are limited to short time scales. As a result, silent functions (e.g., adult functions that are already encoded in the oocyte), embryogenesis, and evolution are not viewed as agent properties. Also, it is questionable if the term "consciousness" can be applied at the level of vegetative semiosis.

Figure 1. Major transitions in the evolution of functional information, its components (middle column) and signs (right column).

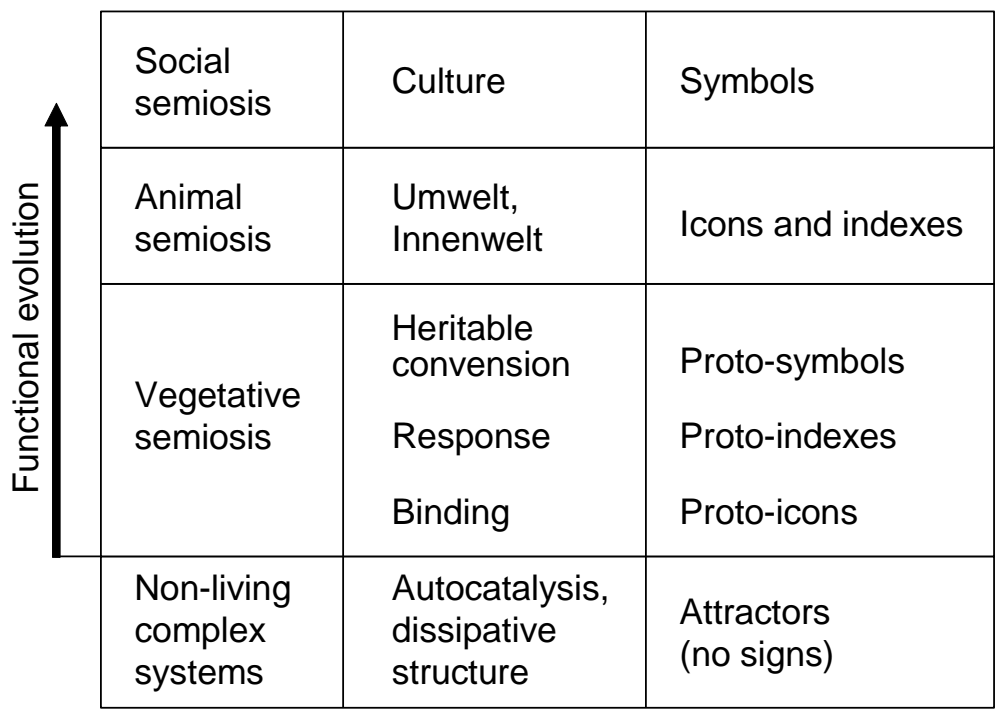

Vegetative semiosis operates at the molecular level and differs substantially from higher levels of semiosis because molecules are recognized and interpreted via direct chemical interactions (e.g., by binding and/or catalysis) rather than by association with ideal representations of objects. The difference is so profound that Umberto Eco decided to "exclude from semiotic consideration neurophysiological and genetic phenomena" [42]. However, incorporation of molecular processes into semiotics opens new horizons in our understanding of the origin and evolution of life $[13,39,43]$; thus, it cannot be easily dismissed. Moreover, the analysis of molecular signs can help us to understand the origin and nature of human signs.

The key feature of vegetative semiosis is code-based mapping [19]. Most common examples of code-based mapping involve adaptors, such as tRNA in protein synthesis and surface receptors in signal transduction [19,24]. In both examples, adaptors connect two entirely unrelated kinds of molecules. The notion of code-based mapping can be extended even to reflexive relations (e.g., polymerization of actin or tubulin) and binary relations (e.g., specific catalysis). It may be argued that reflexive and binary relations lack the arbitrariness/contingency of the adaptor-mediated code. However, actin is a large molecule and it includes an "internal adaptor" specialized for the function of polymerization. Actin is not a "natural molecule" but a product of adaptive evolution, and therefore it is contingent. Nucleotides are much smaller than proteins, but the mapping of nucleotides A-T and 
C-G in the DNA still can be viewed as a code-based binary relation. Random chemical synthesis can generate a wide variety of nucleobase-like molecules. Yet, only 4 nucleobases are found in the DNA, which indicates that they were selected based on their functionality.

However, code-based mapping alone is not semiosis yet, and therefore, the code-model of semiosis proposed recently by $[13,24]$ seems incomplete. To become semiosis, this code-based mapping needs to be embedded in a functional agent and support the functions of this larger system. Thus, semiosis requires interaction between systems of two (or more) hierarchical levels, which can be taken as an important clue for reconstructing the origin of life. Autocatalysis alone is not semiosis and not life because there is no second level. To become a coding system, autocatalytic molecules should be able to modify (encode) the properties of a their local environment so that the environment becomes more favorable for autocatalysis [44]. This functional linkage is a necessary condition for cooperation between multiple autocatalytic components if they happen to share their local environment. In contrast, unlinked autocatalytic systems can only compete but not cooperate.

Life did not start from heteropolymers like nucleic acids because monomers were not available in sufficient quantities as resources [22]. Even if several nucleotides appeared in close proximity due to a once-in-a-universe lucky coincidence and are then used to synthesize a complimentary RNA strain in a hypothetical protoorganism, there would be no nucleotides left for the reproduction of the next generation. It is also unlikely that life started from large autocatalytic sets of peptides, as proposed by Kauffman [45], because there is no sufficient supply of monomers (i.e., amino acids) to make peptides. The model of random catalysis does not work even if applied to simple organics molecules due to the following problem: most abundant organic molecules (e.g., saturated hydrocarbons) are inert. According to the alternative "coenzyme world" model of the origin of life, small coenzyme-like molecules became autocatalytic on the surface of oil (i.e., hydrocarbon) microspheres in water and modified surface properties of microspheres in a beneficial way [44]. These molecules were called "coding elements" because they replicate via autocatalysis and encode surface properties of microspheres, which together make a heritable metabolism. Such simple systems can further evolve by accumulation of additional coding elements that can help in capturing energy and other resources. Because various kinds of coding elements were not connected, they were transferred to offspring systems in different combinations. Despite random transfer, such combinatorial heredity can be stable because (1) coding elements are present in multiple copies and therefore each offspring has a high probability to get the full set, and (2) natural selection preserves preferentially organisms with a full set of coding elements. The efficiency of the later mechanism was shown in a "stochastic corrector model" [46]. New types of coding elements can be added by (1) recruitment of entirely new molecules from the environment, (2) modification of existing coding elements and (3) polymerization of coding elements or their products [44].

The transition from the "coenzyme world" to the "RNA world" can be seen through the invention of template-based replication. Initial steps of primordial evolution were slow and inefficient because there was no universal rule for producing new coding elements. Some improvement was likely achieved by transformation of old coding elements into novel ones via modification of functional groups or polymerization. However, there has been no streamlined procedure for making new coding elements. Such a procedure was invented in the form of a template-based (or digital) replication. It is a special 
case of autocatalysis, where each coding element is a linear sequence made of a few kinds of monomers, and copying is done sequentially via predefined actions applied to each monomer [46]. Digital replication makes the coding system universal because the algorithm works for any sequence; hence, there is no need to invent recipes for copying modified coding molecules. The starting point for the origin of template-based replication is the existence of polymers with either random or repetitive sequence [44]. Polymers may initially stick to each other to perform some other functions, e.g., to increase stability and facilitate polymerization. The shorter strand of the paired sequence can then be elongated by adding monomers that weakly match to the overhanging longer strand. Then natural selection would have supported the increase of specificity of this process and helped to produce better copies of existing polymers. Some coding elements became specialized in assisting the process of template-based replication playing the role of polymerase; as a result, the replication process became passive. Invention of digital replication, therefore, may have been the turning point in the origin of life, which supported unlimited hereditary potential $[44,47,48]$ and caused a rapid increase in the abundance and complexity of coding elements.

Although the rate of processes in primordial organisms depended on the environment, these effects initially were not regulated by organisms. Regulation requires sensors, which are adaptor molecules that mediate the effects of environment on organism functions. In a simple case, sensors interact directly with functional modules. For example, many bacterial transcription factors are adaptors connecting the promoter of a regulated gene and a ligand (i.e., signaling molecule) [49]. In a more complex case, sensors generate small short-lived molecules (messengers) after ligand binding, and then messengers travel to functional modules (e.g., promoters) and modify their activity. Production of sensors have to be a heritable function before any sensing occurs. Thus, we can hypothesize that heredity was the most ancient function of primordial signs, and sensing appeared later when organisms mastered the production of complex molecules which can play the role of sensors.

As the number of sensors/adaptors increased in the evolution of organisms, they became integrated into sensory networks via logic gates. For example, transcription of a gene is often activated by simultaneous binding of two or more transcription factors to neighboring DNA motifs in the promoter. The combination of multiple DNA motifs makes a composite sensor, which works as the "AND" logic gate. The mRNA molecule is another example of the "AND" logic gate because it combines multiple sets of nucleotide triplets. These triplets bind sequentially to tRNA adapters within the ribosome and control the order of amino acids in the synthesized protein. The "OR" gate is simply a sensor that can respond to multiple external signs. By using logic gates, organisms can generate messengers that represent complex conditions. Logic gates are encoded either as direct prescriptions for signal processing (e.g., cis-regulatory modules in promoters), or as prescriptions for making proteins that play the role of gates. This innate logic evolves via genetic selection only; thus, it cannot be modified within the life span of an organism if to some reason it stops producing beneficial effects. To overcome this limitation, organisms developed epigenetic mechanisms for modifying logic gates on demand. For example, a gene with multiple cis-regulatory modules in its promoter may initially carry open chromatin at all locations. However, after some "memory triggering" event, the chromatin becomes condensed at all modules except the one that was functional at the time of the event. This can be viewed as a primitive mechanism of learning $[19,40]$. 
According to Kull, vegetative semiosis is based solely on iconic sign relations because code-based matching (or "recognition") is analogous to visual recognition of icons [19]. Semiotic notions of Peirce are highly generic, and binding of signal molecules may indeed match his definition of icon: "An icon is a sign fit to be used as such because it possesses the quality signified." [15]. However, it is important to keep in mind a tremendous gap that separates human icons (e.g., visual shapes or sound tunes) from molecular interactions that lack any elements of perception or classification. Human icons are associated with ideal representations of objects, which are not yet present at the molecular level. Because vegetative semiosis is so different from animal and social semiosis, I suggest to use prefix "proto" for semiotic terms applied at the vegetative level. Examples of proto-icons include both simple molecular bindings (e.g., binding of cAMP to a CAP receptor) and complex multi-component bindings (e.g., complementary binding of nucleic acids in DNA replication, transcription, and miRNA binding).

Besides proto-icons, vegetative semiosis includes proto-indexes and proto-symbols. Here I disagree with the idea of Kull [19] that vegetative semiosis is solely iconic. For example, an adaptor molecule is a proto-index because it has two binding surfaces for interaction with two different kinds of molecules, which are physically connected. Proto-indexes often form chains of signal transduction which transfer signals detected at the cell membrane to the nucleus. Proto-symbols are conventional sign relations established for vertical and/or horizontal communication. For example, DNA plays the role of a protosymbol in a sequence of processes that start from mRNA synthesis on the DNA template and ending with protein synthesis on the mRNA template. Protein synthesis follows the rule of the genetic code which is a heritable "natural convention" based on a set of molecular adapters (tRNAs and acyl-transferases) [13].

Proto-symbols are components of the genetic proto-language which appeared much earlier in evolution than animal and human languages. This proto-language gradually changed along evolutionary lineages. The structure of the mammalian genome is entirely different from the bacterial genome, although some features are highly conserved and remained almost unchanged through billions of years (e.g., the genetic code table). The major changes appeared in the transitions from prokaryotes to eukaryotes. These include the development of additional code systems: the chromatin code, splicing code, miRNA code, and others [24]. The transition from unicellular to multicellular organisms was associated with the emergence of complex signaling between cells. Cis-regulatory regions in promoters evolved from simple combinations of transcription factor binding motifs to complex ensembles that control the function of enhanceosomes.

\section{Animal and Social Semiosis}

As the number of perceived signals increased in evolution, agents learned how to integrate them into meaningful categories representing various objects and situations (e.g., food items, partner agents, and enemies) and predict events using models. These classifications and models represent the knowledge of an agent about itself and its environment. Following the terminology of Uexküll, this knowledge is the Innenwelt and Umwelt of the organism [2]. Although Kull assumes that Umwelt may exist even at the vegetative level of semiosis [19,50], I prefer to limit the use of this term to the higher levels of semiosis. At the vegetative level, signs are mere prescriptions of actions and do not carry knowledge. In contrast, signs at the animal and social levels of semiosis are linked with ideal 
representations of objects, situations, and actions. However, higher levels of semiosis are grounded at the vegetative level because all activities of an organism (e.g., sensing, movement, and interpretation) are apparently supported by certain molecular functions, although we still know very little about molecular mechanisms of neural processes. Information processed by animal and social semiosis does not necessary induce physical actions, however it still can be called "functional information" because: (1) it involves mental functions (e.g., accumulation of knowledge) and (2) may affect future physical actions.

Animal semiosis include icons (e.g., visual images and sound patterns), and indexes, which are associations between classes of objects, as well as between classes of objects and actions [19]. The knowledge about objects, their associations, and possible modifications (Umwelt) is stored in the individual memory but it is not transmitted to other animals because animals do not have the language capacity. Only humans fully crossed the transition zone from animal to social semiosis and developed symbolic languages for efficient horizontal communication [19]. Birds and mammals can use a few symbols, but these seem to be limited to a small number of biological functions (e.g., danger warnings and sexual courtship).

Individual memory is not heritable, and thus, the Umwelt cannot be transferred genetically to the next generation. However, development of Umwelt can be strongly constraint by heritable features of the body. Because organs are animal tools, this body-mind link is analogous to the law of the instrument, which states that "When the only tool you have is a hammer, it is tempting to treat everything as if it were a nail" [51]. In addition to effectors (legs, tails, mouth), sense organs are highly important as constraints for the developing Umwelt. The structure, sensitivity, and resolution of senses determines what patterns an animal will be able to learn in its individual life. The size of the body, life span, and movement speed also contribute to the perception and interpretation of the world. Thus, animals of different sizes (e.g., a cow and ant) perceive the same environment (meadow) in entirely different ways [2]. Because of these heritable constraints, Umwelten of the progeny appear very similar to the Umwelten of their parents (if they remain in the same habitat) even in the absence of inter-generational communication. The ability of animals to classify and model external objects emerges through recursive sensory-motor operations, and therefore, stable cognitive states can be characterized as eigenvectors or eigenbehaviors [8,52]. The cognitive dynamic system depends on heritable neural architecture; thus eigenbehaviors can be well reproduced between generations. But environmental factors also affect the dynamics both directly during the morphogenesis of the brain and indirectly through the signals from sense organs. As a result, eigenbehaviors are flexible enough and can be adjusted to the changing environment.

In contrast to Umwelt, the Innenwelt has a substantial heritable component in organisms. Organisms need robust methods for producing body parts, organs, and tissues, and these methods should remain functional in variable environments as well as in variable conditions within the body over long evolutionary times. Adaptive evolutionary changes in one organ should not affect the methods for generating other organs, because otherwise the species may lose its adaptability. High robustness can be achieved only by functional modularity, which means isolating a given functional sub-network from external effects [53]. This can be achieved by passive isolation (breaking signaling links) as well as by active compensatory responses within the sub-network. Functional modules can be compared with 
encapsulated objects in object-oriented programming languages (e.g., C++ or Java). Programmed objects have pre-determined inputs and outputs and cannot crash even if other parts of the code contain errors. Similarly, developmental modules are mostly independent from other processes but can be initiated by pre-determined specific factors (e.g., growth factors or genes). Manipulations of these factors may induce organs in unusual places. For example, the antennapedia mutation of Drosophila transforms antennae into legs [54]. Developmental modules can be viewed as an ideal representations of future organs because the organ is already "drafted" and it takes a single switch to initiate its construction. These ideal representations are similar to mental representations; and thus, I view developmental modules as components of animal semiosis rather than vegetative semiosis.

Considering levels of vegetative, animal, and social semiosis, where can we place the semiosis of programmed artificial devices? As components of human semiosis, they belong to the social symbolic level. However, if taken alone artificial devices can operate only at vegetative and animal level. Because most machines cannot learn and evolve, their functional information has human origin. Even computer viruses, which is the only class of autopoietic artificial systems, are not capable of adaptive evolution. Machines can process icons and indexes supplied by humans, but most of them do not develop their own icons and indexes. However, there are trainable computers which can learn to classify objects or optimize actions for reaching certain goals [11]. They can find non-trivial solutions and adapt to situations unforeseen by designers. If such machines are supplied with both sensors and effectors then, theoretically, they can start developing new sequences of actions for reaching certain goals. However humans have not made any artificial agents capable of autonomous adaptive evolution. Possibly, this kind of agents will be created by imitating living organisms, a strategy known as synthetic biology [55,56]. A major step towards creation of synthetic organisms was the synthesis of a bacterial chromosome by Venter [57].

\section{In search for a New Philosophy of Science}

Cybernetics and biosemiotics deviate substantially from the old paradigm of science, which is based on objective observations and experiments. Agents are subjective beings, and it is not possible to understand them well in pure objective notions. Our knowledge on the goals, perceptions, and possible actions of agents is limited (except for simple human-made agents), thus our predictions are uncertain, and the value of any control is limited. Objectivism's axiom of identity $(\mathrm{A}=\mathrm{A})$ does not hold with agents because they evolve, learn, and change before our eyes. Thus, if we want to include agents into the sphere of science we need to update the philosophy of science.

An alternative approach to the traditional objectivist epistemology was developed within theories of pragmatism and constructivism. Pragmatism starts the argument from subjective goals and values of agents: "truth is that which works" [58]. In particular, knowledge of an agent is "true" if it is useful (i.e., helps to achieve goals). Constructivism is similar to pragmatism because it emphasizes the usefulness of knowledge, however it does not consider "truth" and "usefulness" as equivalents. It can be best described by the words attributed to George Box: "all models are wrong but some are useful". According to radical constructivism, knowledge is constructed rather than discovered from the world [14]. Different organisms and species develop their own models of reality; thus, according to Maturana, 
the universe turns into a multiverse [59]. This notion of multiverse is consistent with the idea of Uexküll that organisms develop and use different models of their environment (Umwelten) [2].

However, both pragmatism and constructivism have a serious flaw: they underestimate or totally ignore the role of logic as a creative force. Pragmatism has reduced truth to usefulness, and radical constructuvism does not view truth as a useful concept. This prompted Brier to return to the objective idealism of Charles Peirce which included "synechism, an evolutionary perspective, and a pragmatic(istic) epistemology" [14]. Chance (potentiality) and laws (habits of the universe) are assumed to be objective components of the world (its Firstness and Secondness, respectively), and new laws emerge as the universe evolves. Finally, the world includes observers/agents who integrate chances and laws into useful representations and habits (Thirdness). Brier interprets Firstness as "pure feeling" and as a chance in quantum field theory; Secondness is represented by classical kinematics, thermodynamics, resistance, and will; and Thirdness corresponds to chemical sciences. On the top of these three levels of ontology Brier proposed to consider two additional levels: the fourth level corresponds to living organisms capable of semiotic interactions, and the fifth level belongs to human language and rationality [14].

Objective idealism provides the comfort of objectivity of human knowledge, however it has some problems. First, physical laws are models of nature developed by agents (as it is assumed by constructivism), rather that parts of the nature itself. Models reflect real regularities in nature, but they are not equivalent to these regularities. Models are proposed, tested, and get accepted by agents if they provide useful and reliable results. However, they are discarded if better models (i.e., simpler, more accurate, and applicable in a broader area) become available. Also, new models have almost nothing in common with previous models [60]. For example, the string theory has no similarity to the Newton's physics. Second, theoretical models are formulated using abstract notions (e.g., material point, ideal gas), and their application to the real world requires abstraction (i.e., separation of important information from non-important). Then why to consider these models as a part of the world if they cannot be applied directly to the world? Of course, objective idealism can assume that all possible abstractions are also components of the world. But if the universe is capable of making abstractions, then it should have some sort of mind, an idea which is not well compatible with science. According to evolutionary biology, mind and consciousness appeared at the latest stages of evolution in animals with well-developed nervous system, and there is no evidence that mind can exist in non-living or non-material systems. Brier uses Peirce's triadic philosophy, which considers chaos and pure feeling as the primary ontological level of the universe [14]; however, molecular biology shows that sensing and feeling is a property of organisms with receptors. Feelings emerged in evolution at the time when primitive organisms discovered how to manufacture and use receptors. Because of these problems, objective idealism of Peirce, as presented by Brier, is not likely to be accepted as a philosophy of science.

An alternative way of combining constructivism and pragmatism with logic is to view logic as a useful tool that facilitates creative evolution of communicating agents [36]. In organisms, logic is used to derive novel behaviors that are more likely to be successful than behaviors selected by chance. At the level of knowledge, logic is used to derive new statements that are more likely to be true than randomly assembled statements. In mathematical logic, derived statements are always true if they are 
based on true initial statements (axioms). Self-organization and eigenbehavior are examples of internal logic in agents. Self-organization rules determine what modifications of organs are possible within short-term individual development or long-term evolution. For example, certain rules in the evolution of plant leaves were identified by Meyen [61]. Similarly, not all possible distinctions in the environment can be detected by autonomous agents, but only those that correspond to eigenbehaviors [62].

The usefulness of logic belongs to the meta-level compared to the usefulness of actions (i.e., it represents adaptability rather than adaptation), and it is selected at the time scales of macroevolution. In particular, rules of self-organization and eigenbehavior are subject to selection at the level of lineages rather than individual organisms because some of these rules yield higher adaptability and broader diversification within certain lineages $[63,64]$. The usefulness of logic is more related to its internal organization rather than to its interface with actions. Thus, humans often use aesthetic criteria to select logical systems based on their universality, simplicity, and richness.

In contrast to utility-oriented pragmatism and logic-oriented logical positivism, I assume that the evolution of semiotic systems is driven by two interdependent factors: utility and logic [36]. Logic determines possible or preferred directions of change, and utility helps to find working solution among these possibilities. However, the usefulness of logical rules is limited to the set of routinely communicating agents, which I called "communication system" [36], and therefore it provides only local objectivity. The world has certain regularities, however each kind of agents perceives different sets of these regularities. Two communication systems may have partially overlapping ontologies, and this overlap allows them to establish limited communications. But it is hardly possible to develop a universal ontology that covers all regularities of the world because such ontology would be infinite, and hence, not operational (because of infinite processing time). This worldview can be called "local realism" because the knowledge about the world is shared locally within a certain communication system. In rare cases, it can be transferred to another communication system with similar logic and ontology. But there is no way to transfer knowledge between entirely unrelated communication systems (e.g., we cannot explain to an ant that "the snow is white"). According to this worldview, scientific knowledge is objective locally, i.e., in relation to the communication system of science with its current rules of experiment design, statistical inference, and theoretical generalization. However, these rules evolve as science expands, and any change prompts for revisions in the whole volume of scientific knowledge. Local realism is compatible with the scientific method, but it also allows the existence of alternative ontologies in other communication systems (e.g., in religions and local cultures).

\section{Conclusions}

The synthesis of biosemiotics and cybernetics is seen as integration of their efforts in the study of agents, a new cross-disciplinary ontological entity. First, the focus of biosemiotics should be shifted from living organisms to agents in general, which all belong to the pragmasphere or functional universe. Second, agents need to be considered in the context of their hierarchy and origin because their semiosis can be inherited from parental agents or induced by higher-level agents. In particular, the absence of learning in isolated individual agents does not mean that they are not semiotic. Third, the cybernetics needs to shift from the computational paradigm to the functional paradigm. I believe that the notion of functional information, which is a set of signs used by agents to encode and control their 
functions, can be a starting point for this transition. And fourth, following the evolutionary principles, multiple levels of semiosis should be distinguished to classify molecular agents, cells, plants, animals, humans, and machines. The lowest vegetative levels of semiosis is the most intriguing, as it provides clues for the origin of life, and also serves as a basis for the emergence of animal, and social levels of semiosis. Vegetative semiosis differs substantially from higher levels of semiosis because signs are recognized and interpreted via direct code-based matching and are not associated with ideal representations (models and classifications) of objects. Due of these differences, I consider a separate classification of signs at the vegetative level, which includes proto-icons, proto-indexes, and proto-symbols. Higher levels of semiosis which include classifications, models, and logic are grounded in the vegetative semiosis.

\section{Acknowledgements}

I would like to thank Kalevi Kull, Tartu University, for inspiring this work; and Søren Brier, Copenhagen Business School, for valuable comments on the philosophic foundations of cybersemiotics. This work was supported by the Intramural Research Program of the National Institute on Aging, NIH.

\section{References and Notes}

1. Schrödinger, E. What is life? The Physical Aspect of the Living Cell; Cambridge University Press: Cambridge, UK, 1940.

2. Uexküll, J. The theory of meaning. Semiotica 1982, 42, 25-82.

3. Wiener, N. Cybernetics or Control and Communication in the Animal and the Machine; Wiley \& Sons Inc.: New York, NY, USA, 1948.

4. Shannon, C.E. A mathematical theory of communication. Bell Syst. Tech. J. 1948, 27, 379-423, 623-656.

5. Sebeok, T.A. Biosemiotics: its roots, proliferation and prospects. Semiotica 2001, 134, 61-78.

6. Brazhnik, O.; Jones, J.F. Anatomy of data integration. J. Biomed. Inform. 2007, 40, 252-269.

7. Heylighen, F.; Joslyn, C. Cybernetics and second-order cybernetics. In: Encyclopedia of Physical Science and Technology; Meyers, R.A., Ed.; Academic Press: New York, NY, USA, 2001; pp. 1-24.

8. von Foerster, H. Understanding: Essays on Cybernetics and Cognition; Springer: New York, NY, USA, 2003.

9. Maturana, H.; Varela, F. Autopoiesis and Cognition: the Realization of the Living; D. Reidel Publishing Co.: Dordrecht, The Netherlands, 1980.

10. Emmeche, C. The Garden in the Machine: The Emerging Science of Artificial Life; Princeton University Press: Princeton, NJ, USA, 1994.

11. Cariani, P. Towards an evolutionary semiotics: the emergence of new sign-functions in organisms and devices. In Evolutionary Systems; Vijver, G.V.; Salthe, S.; Delpos, M., Eds.; Kluwer: Dordrecht, The Netherlands, 1998; pp. 359-377.

12. Sharov, A.A. Biosemiotics: functional-evolutionary approach to the problem of the sense of information. In Biosemiotics. The Semiotic Web 1991; Sebeok, T.A., Umiker-Sebeok, J., Eds.; Mouton de Gruyter: New York, NY, USA, 1992; pp. 345-373. 
13. Barbieri, M. The Organic Codes: an Introduction to Semantic Biology; Cambridge University Press: Cambridge, UK/New York, NY, USA, 2003.

14. Brier, S. Cybersemiotics: Why Information Is Not Enough; Toronto University Press: Toronto, Canada, 2008.

15. Peirce, C.S. The Essential Peirce: Selected Philosophical Writings; Indiana University Press: Bloomington, IN, USA, 1998.

16. Collier, J. Causation is the transfer of Information. In Causation and Laws of Nature; Sankey, H., Ed.; Kluwer: Dordrecht, The Netherlands, 1999; pp. 279-331.

17. Brillouin, L. Science and Information Theory. Academic Press: New York, NY, USA, 1962.

18. Burgin, M. Super-recursive Algorithms; Springer: New York, NY, USA, 2005.

19. Kull, K. Vegetative, animal, and cultural semiosis: the semiotic threshold zones. Cogn. Semiotic. 2009, 4, 8-27.

20. Rosen, R. Dynamical System Theory in Biology; Wiley-Interscience: New York, NY, USA, 1970.

21. Rashevsky, N. Mathematical Biophysics; University of Chicago Press: Chicago, IL, USA, 1938.

22. Sharov, A.A. Genetic gradualism and the extraterrestrial origin of life. J. Cosmol. 2009, 5, 833-842.

23. Hoffmeyer, J. Biosemiotics: an Examination into the Signs of Life and the Life of Signs; University of Scranton Press: Scranton, PA, USA, 2008.

24. Barbieri, M. Is the cell a semiotic system? In Introduction to Biosemiotics. The New Biological Synthesis; Barbieri, M., Ed.; Springer: Dordrecht, The Netherlands, 2008; pp. 170-207.

25. Gould, S.J.; Eldredge, N. Punctuated equilibria: the tempo and mode of evolution reconsidered. Paleobiology 1977, 3, 115-151.

26. Dawkins, R. The Blind Watchmaker; Norton: New York, NY, USA, 1986.

27. Weber, B.H. On the emergence of living systems. Biosemiotics 2009, 2, 343-359.

28. Sharov, A.A. Genome increase as a clock for the origin and evolution of life. Biol. Direct. 2006, $1,17$.

29. Consortium, G.O. The Gene Ontology project in 2008. Nucleic. Acids. Res. 2008, 36, D440-D444.

30. Vo, N.; Goodman, R.H. CREB-binding protein and p300 in transcriptional regulation. J. Biol. Chem. 2001, 276, 13505-13508.

31. Ahmadjian, V. Tribouxia: Reflections on a perplexing and controversial lichen photobiont. In Symbiosis. Mechanisms and Model Systems; Seckbach, J., Ed.; Kluwer: Dordrecht, The Netherlands, 2002; pp. 375-383.

32. Weber, N.A. Gardening Ants, the Attines; American Philosophical Society: Philadelphia, PA, USA, 1972.

33. Klepzig, K.D.; Moseri, J.C.; Lombarder, F.J.; Hofstetter, R.W.; Ayres, M.P. Symbiosis and competition: complex interactions among beetles, fungi and mites. Symbiosis 2001, 30, 83-96.

34. Turchin, V.F. The Phenomenon of Science. Columbia University Press: New York, NY, USA, 1977.

35. Smith, J.M.; Szathmáry, E. The Major Transitions in Evolution; W.H. Freeman/Spektrum, Oxford: New York, NY, USA, 1995. 
36. Sharov, A.A. Role of utility and inference in the evolution of functional information. Biosemiotics 2009, 2, 101-115.

37. Hoffmeyer, J.; Emmeche, C. Code-duality and the semiotics of nature. In On Semiotic Modeling; Anderson, M.; Merrell, F., Eds.; Mouton de Gruyter: Berlin, Germany/New York, NY, USA, 1991; pp. 117-166.

38. Danesi, M.; Perron, P. Analyzing Cultures: an Introduction and Handbook; Indiana University Press: Bloomington, IN, USA, 1999.

39. Witzany, G. Biocommunication and Natural Genome Editing; Springer: New York, NY, USA, 2009.

40. Bateson, G. Steps to an Ecology of Mind; Collected Essays in Anthropology, Psychiatry, Evolution, and Epistemology; Chandler Pub. Co.: San Francisco, CA, USA, 1972.

41. Arrabales, R.; Ledezma, A.; Sanchis, A. ConsScale: A pragmatic scale for measuring the level of consciousness in artificial agents. J. Conscious. Stud. 2010, 17, 131-164.

42. Eco, U. A Theory of Semiotics; Indiana University Press: Bloomington, IN, USA, 1976.

43. Pattee, H.H. How Does a Molecule Become a Message? Dev. Biol. Supp. 1969, 3, 1-16.

44. Sharov, A.A. Coenzyme autocatalytic network on the surface of oil microspheres as a model for the origin of life. Int. J. Mol. Sci. 2009, 10, 1838-1852.

45. Kauffman, S.A. Autocatalytic sets of proteins. J. Theor. Biol. 1986, 119, 1-24.

46. Szathmáry, E. The first replicators. In Levels of Selection in Evolution; Keller, L., Ed.; Princeton University Press: Princeton, NJ, USA, 1999; pp. 31-52.

47. Jablonka, E.; Szathmáry, E. The evolution of information storage and heredity. Trends Ecol. Evol. 1995, 10, 206-211.

48. Szathmáry, E. The origin of replicators and reproducers. Philos. Trans. R. Soc. Lond. B Biol. Sci. 2006, 361, 1761-1776.

49. Rigali, S.; Schlicht, M.; Hoskisson, P.; Nothaft, H.; Merzbacher, M.; Joris, B.; Titgemeyer, F. Extending the classification of bacterial transcription factors beyond the helix-turn-helix motif as an alternative approach to discover new cis/trans relationships. Nucleic. Acids. Res. 2004, 32, 3418-3426.

50. Kull, K. Umwelt. In The Routledge Companion to Semiotics; Cobley, P., Ed.; Routledge: Ney York, NY, USA, 2010; pp. 348-349.

51. Maslow, A.H. Psychology of Science. A Reconnaissance. Harper: New York, NY, USA, 1966.

52. Rocha, L.M. Eigenbehavior and symbols. Syst. Res. 1996, 13, 371-384.

53. Schlosser, G.; Wagner, G.P. Introduction: The modularity concept in developmental and evolutionary biology. In Modularity in Development and Evolution; Schlosser, G., Wagner, G.P., Eds.; University of Chicago Press: Chicago, IL, USA/London, UK, 2004; pp. 1-11.

54. Gibson, G. Insect evolution: Redesigning the fruitfly. Curr. Biol. 1999, 9, R86-R89.

55. Cao, H.; Romero-Campero, F.J.; Heeb, S.; Camara, M.; Krasnogor, N. Evolving cell models for systems and synthetic biology. Syst. Synth. Biol. 2010, 4, 55-84.

56. Basu, S.; Gerchman, Y.; Collins, C.H.; Arnold, F.H.; Weiss, R. A synthetic multicellular system for programmed pattern formation. Nature 2005, 434, 1130-1134. 
57. Gibson, D.G.; Benders, G.A.; Andrews-Pfannkoch, C.; Denisova, E.A.; Baden-Tillson, H.; Zaveri, J.; Stockwell, T.B.; Brownley, A.; Thomas, D.W.; Algire, M.A.; Merryman, C.; Young, L.; Noskov, V.N.; Glass, J.I.; Venter, J.C.; Hutchison, C.A.; Smith, H.O.; Complete chemical synthesis, assembly, and cloning of a Mycoplasma genitalium genome. Science 2008, 319, 1215-1220.

58. James, W. Pragmatism, A New Name for Some Old Ways of Thinking, Popular Lectures on Philosophy; Longmans, Green, and Co.: New York, NY, USA, 1907.

59. Maturana, H.R. The biological foundation of self-consciousness and the physical domain of existence. In Physics of Cognitive Process; Caianiello, E.R., Ed.; World Scientific: Singapore, 1987; pp. 324-379.

60. Kuhn, T.S. The Structure of Scientific Revolutions. University of Chicago Press: Chicago, IL, USA, 1970.

61. Meyen, S.V. Fundamentals of Palaeobotany. Chapman and Hall: London, UK, New York, NY, USA, 1987.

62. Rocha, L.M. Selected self-organization. In Evolutionary Systems: Biological and Epistemological Perspectives on Selection and Self-organization; Salthe, S., Van de Vijver, G., Delpos, M., Eds.; Kluwer Academic Publishers: Dordrecth, The Netherlands, 1998; pp. 341-358.

63. Eldredge, N. Unfinished Synthesis: Biological Hierarchies and Modern Evolutionary Thought; Oxford University Press: New York, NY, USA, 1985.

64. Depew, D.J.; Weber, B.H. Darwinism Evolving: Systems Dynamics and the Genealogy of Natural Selection; MIT Press: Cambridge, MA, USA, 1995.

(C) 2010 by the authors; licensee MDPI, Basel, Switzerland. This article is an open-access article distributed under the terms and conditions of the Creative Commons Attribution license (http://creativecommons.org/licenses/by/3.0/). 